draw attention to the negative studies (Barr et al, 1991; Ishida, 1993; Wang et al, 1993; Curtis et al, 1993) reported for pseudoautosomal linkage in schizophrenia which Crow et al did not cite.

References appear below

D. Curtis

G. KALSI

Molecular Psychiatry Laboratory

H. M. D. GURLING

Department of Psychiatry

University College London Medical School

London WIP 6DB

AUTHORs' REPLY: In responding to Curtis et al we make three points.

First, we note that they misrepresent out paper:

(a) by stating that we did not refer to the paper on linkage by Wang et al when this paper is cited in the Discussion (p. 163)

(b) by implying that we failed to refer to two abstracts (Curtis et al, Ishida et al) which in fact were presented and published after our paper was accepted (these studies are apparently in agreement with our conclusion that the evidence does not support a locus within the pseudoautosomal region; Curtis et als last sentence leaves us in doubt whether they have understood that, as stated in our summary and Discussion, this is a major conclusion of our study)

(c) by suggesting that the diagnostic criteria we have used are not specified when these are stated (p. 160)

Second, we respond to what we take to be Curtis et $a$ 's main point, that the positive lod score can be accounted for by allele sharing on the Y chromosome at MIC2. We are in agreement with this and state (p. 162) that "it is likely that the lod score of 2.44 is accounted for by linkage on the Y chromosome, i.e. to sex".

Could this finding be due, as Curtis et al suggest, to multiple testing or to a predominance of affected males? We investigated how likely it would be that the lod scores we observed had arisen by chance (assuming an autosomal gene for schizophrenia) with our sample and method of analysis. We simulated a marker (MIC2) that was unlinked to the phenotype (schizophrenia) but linked to the boundary of the pseudoautosomal region (sex locus). The linkage between MIC2 (using information from the p19b-TaqI probe ${ }^{1}$ ) and the sex locus (in males

1. The SLINK program was used for the simulations. Only the TaqI information was used because it was difficult to simulate all three probes together in this program. $r=0.05$, in females $r=0.02$ ) was as expected for the pseudoautosomal region and as estimated from our data.

The simulated pedigrees were then analysed with ISIM (using the ILINK algorithm) and maximum lod scores were calculated over a range of male and female recombination fractions. As in our paper, we used a lifetime penetrance of 0.5 , penetrance for phenocopies of 0.005 , and a gene frequency of 0.0052 . The overall maximum of $Z=0.5$ occurred at a recombination fraction of 0.15 in males and approximately 0.5 in females. This suggests that the maximum lod scores found in the analysis should be corrected by 0.5 . Using data for the pl9b-TaqI probe for MIC2 alone (as in the simulation) we find a maximum lod score $(Z)$ of 2.95 , giving 2.45 after correction. These calculations suggest that our lod score of 2.4 cannot be accounted for by "artefactual evidence in favour of linkage when there is an excess of affected sibling pairs who are concordant for sex".

In addition, the sample was selected for schizophrenia and not for sex. More of our probands are males than females ${ }^{2}(135: 51)$ - probably because our sample is weighted towards early onset. Whether Curtis et al are justified in describing this as an 'excess' is at the heart of the matter. The issue, as we see it, is whether the well known sex differences in schizophrenia (e.g. with respect to age of onset) are extrinsic to the disease process or whether they reflect directly on its genetic origin. We refer interested readers to our original paper (Crow et al, 1989) and to the subsequent discussion (Curtis \& Gurling, 1990; Crow et al, 1990), of which we do not altogether share Curtis et als interpretation.

Third, we agree with Curtis et al that a lod score of 2.44 is no more than suggestive evidence of linkage. Like all such findings it requires further investigation, by ourselves (e.g. DeLisi et al, 1994) and others. The point is justly made by the group that reported a lod score of 6.49 to a locus on the long arm of chromosome 5 (Sherrington et al, 1988), a finding unsupported by subsequent studies.

Barr, C. L., Kennedy, J. L. \& Pakstis, J. (1991) Progress in genome scan for linkage in schizophrenia. Psychiatric Genetics, 2, 66 .

Collinge, J. S., Delisi, L. E. \& Boccio, A. (1991) Evidence for a pseuoautosomal locus for schizophrenia using the method of affected sib pairs. British Journal of Psychiatry, 158, 624-629.

2. Out of a total of 85 families studied there were $38 \mathrm{MM}, 8 \mathrm{FF}$ and $28 \mathrm{MF}$ pairs. In addition there were 1 FFM, 3 MMF, 4 MMM, I FFMM, $1 \mathrm{MMMM}$, and 1 MMMMMM sets of ill siblings. 
Crow, T. J., Delisl, L. E. \& Johnstone, E. C. (1989) Concordance by sex in sibling pairs with schizophrenia is paternally inherited. Evidence for a pseudoautosomal locus. British Journal of Psychiatry, 155, 92-97.

-, - \& - (1990) In reply ... a locus closer to the telomere? British Journal of Psychiatry, 156, 416-420.

CURTIS, D. \& GuRLng, H. M. D. (1990) Unsound methodology in investigating a pseudoautosomal locus in schizophrenia. British Journal of Psychiatry, 156, 415-416.

KALSI, G., BRYNJOLfSSON, J., RRAD, T., et al (1993) Investigation by linkage analysis of the XY chromosomal region in the genetic susceptibility to schizophrenia. Psychiatric Genetics, 3, 126.

Delist, L. E., Devoto, M., Lofrhouse, R., et al (1994) Search for linkage to schizophrenia on the $\mathrm{X}$ and $\mathrm{Y}$ chromosomes. Neuropsychiatric Genetics (in press).

IsfiDA, T., YonedA, H., SAKA, T., et al. (1993) Pseudoautosomal region in schizophrenia: sex concordance of the affected sibpairs and the association study with DNA markers. American Journal of Medical Genetics, 48, 151-155.

Shirrrington, R., Brynolfsson, J., Peterson, H., et al (1988) Localization of a susceptibility locus for schizophrenia on chromosome 5. Nature, 336, 164-167.

Wang, Z. W., Black, D., Andrensen, N., et al (1993) Pseudoautosomal locus for schizophrenia excluded in 12 pedigrees. Archives of General Psychiatry, 50, 199-204.

Clinical Research Centre

T. J. CRow

Division of Psychiatry

Watford Road

Harrow HAl 3 UJ

New York State Psychiatric Institute

T. LEHNER

State University of New York

L. E. DeLisI

\section{Obstetric complications in schizophrenia}

SIR: Günther-Genta et al (BJP, February 1994, 164, 165-170) found an excess of obstetric complications (OCs) in schizophrenic patients when compared with siblings, normal controls, or other patients. As in most such studies (Lewis, 1989) their findings reveal differences in the main at a low level of statistical significance.

As they point out, their sampling of schizophrenic in-patients leads to a selection bias towards chronicity, and they suggest that the only way of avoiding such bias would be a community study. In our community study (McCreadie et al, 1992) we failed to find a difference between schizophrenic patients and their siblings in their history of OCs. Our community of schizophrenic patients contains some who have had fewer admissions and probably a better prognosis.

Günther-Genta et al question the validity of studies which rely on maternal recall as the source of information on OCs, but O'Callaghan et al (1990) have shown that maternal recall is reliable, and using maternal recall found a rate of definite OCs in their schizophrenic population of $33 \%$, which is comparable to the $45 \%$ found in GüntherGenta et al's studies and which is close to the $35 \%$ that we found. Günther-Genta et al draw attention to the low rates of definite OCs found in Lewis et al's study (1989) (with 'definite' complications in $17 \%$ of schizophrenics and $8 \%$ of controls); they suggest this reflects a low sensitivity of maternal recall. In fact these figures were drawn from information obtained solely from psychiatric records, which will clearly underestimate the proportion of patients with complications and only indirectly reflect the accuracy or otherwise of maternal recall.

LEWIS, S. W. (1989) Congenital risk factors for schizophrenia. Psychological Medicine, 19, 5-13.

-, Owen, M. G. \& Murray, R. M. (1989) Obstetric complications in schizophrenia: methodology and mechanisms. In Schizophrenia: Scientific Progresses (eds S.C. Schultz \& C.A. Tamminga), pp. 56-58. New York: Oxford University Press.

MCCreadie, R. G., Hall, D. J., Berrry, I., et al (1992) Nithsdale schizophrenia surveys. X: Obstetric complications, family history and abnormal movements. British Journal of Psychiatry. 160, 799-805.

O'Callaghan, E. Larkin, C. \& Waddington, J. L. (1990) Obstetric complications in schizophrenia: validity of maternal recall. Psychological Medicine, 20, 89-94.

Department of Psychiatry

David J. Hall Southern General Hospital Glasgow G51 4TF

Crichton Royal Hospital Dumfries

Robin G. McCREAdIE

\section{Early responses to electroconvulsive therapy}

SIR: Rodger et al (BJP, January 1994, 164, 106109) draw attention to the important question of the speed of response to electroconvulsive therapy (ECT). This prompted me to review data from a previous study of ECT and pterin metabolism (Anderson et al, 1992). The original protocol required all subjects to be assessed after two ECT applications, although these data were not reported.

Subjects met DSM-III criteria for major depression with melancholia or psychosis (American Psychiatric Association, 1980). ECT was administered twice weekly using bilateral electrode placement and an Ectron 2 Series ECT device. Severity of depression was measured by the Hamilton Rating Scale for Depression (HRSD; Hamilton, 1969) and the Montgomery-Asberg Depression Rating Scale (MADRS; Montgomery \& Asberg, 1979), but only 